\title{
Proteasas extracelulares producidas por bacterias marinas aisladas de aguas contaminadas con efluentes pesqueros
}

\section{Extracellular proteases produced by marine bacteria isolated from sea water contaminated with fishing effluents}

\author{
Tito Sánchez ${ }^{1,3}$, Jorge León ${ }^{1}$, Juan Woolcott ${ }^{2}$, Katherine Arauco ${ }^{3}$
}

Presentado: 14/07/2004

Aceptado: 12/08/2004

\begin{abstract}
Resumen
Un total de 26 cepas de bacterias marinas con actividad proteolítica fueron aisladas de agua de mar contaminadas con efluentes pesqueros; las mismas que se evaluaron en base al crecimiento y formación de halos de actividad en Agar Marino suplementados con caseína al 1\%, pH 8,0 e incubados a $25^{\circ} \mathrm{C}$ por $72 \mathrm{~h}$. Cinco cepas, seleccionadas por presentar los mejores halos de actividad fueron evaluadas a su vez por su crecimiento y producción de proteasas a diferentes concentraciones de $\mathrm{NaCl}$, rangos de temperatura y $\mathrm{pH}$; siendo consideradas finalmente como bacterias halotolerantes, psicrotróficas y alcalófilas moderadas. Estas cepas también fueron evaluadas por su actividad proteolítica específica sobre la caseína, siendo la cepa CM48 (Pseudomonas sp.) la que presentó la mejor actividad específica $(17,38 \mathrm{U} / \mathrm{mg}$ ) a las 72 horas, y seguidas por las cepas CM45 (Alcaligenes sp.) $(12,09 \mathrm{U} / \mathrm{mg})$ y tres cepas de Aeromonas sp. (CM43, CM44 y CM46) con valores de 12,02; 10,07 y $10,10 \mathrm{U} / \mathrm{mg}$ respectivamente.
\end{abstract}

Palabras clave: Bacterias marinas, psicrotróficas, enzimas extracelulares, proteasas.

\section{Abstract}

A total of 26 strains of marine bacteria with proteolytic activity was isolated from sea water contaminated with fishing effluents; they were evaluated in basis of their growth and formation of activity halos in Marine Agar supplemented with casein to $1 \%, \mathrm{pH} 8,0$ and incubated to $25^{\circ} \mathrm{C}$ per 72 hours. Selected five strains were evaluated on the basis of displaying the best halos of activity as well by their growth and production of proteases at different concentrations of $\mathrm{NaCl}$, temperature ranges and $\mathrm{pH}$; being finally considered as moderate halotolerants bacteria, psicrotrophics and alkalophile. These strains also were evaluated by their specific proteolytic activity on the casein, being strain CM48 (Pseudomonas sp.) the one that presented the best specific activity $(17,38 \mathrm{U} / \mathrm{mg})$ at 72 hours, and followed by strain CM45 (Alcaligenes sp.) $(12,09 \mathrm{U} / \mathrm{mg})$ and three strains of Aeromonas sp. (CM43, CM44 and CM46) with values of 12,$02 ; 10,07$ and $10,10 \mathrm{U} / \mathrm{mg}$ respectively.

Keywords: Marine bacteria, psicrotrophics, extracellular enzymes, proteases.

\section{Introducción}

Las bacterias marinas son por su naturaleza psicrotróficas y halotolerantes; poseen procesos metabólicos adaptados a bajas temperaturas y realizan sus actividades en altas con-

\footnotetext{
${ }^{1}$ Laboratorio de Microbiología Ambiental y Biotecnología. Facultad de Ciencias Biológicas, Universidad Nacional Mayor de San Marcos.

Email: Jorge Leon jleonq@unmsm.edu.pe

${ }^{2}$ Laboratorio de Bioorgánica, Facultad de Química e Ingeniería Química, Universidad Nacional Mayor de San Marcos.

${ }^{3}$ Laboratorio de Biotecnología, Facultad de Farmacia y Bioquímica, Universidad Nacional Mayor de San Marcos.
}

centraciones de sales, elevadas presiones hidrostáticas, $\mathrm{pH}$ alcalinos e incluso condiciones anóxicas. Bajo estas condiciones moderadamente «extremas» pueden producir una serie de metabolitos, entre ellas las enzimas extracelulares (Féller et al., 1996). Entre las enzimas extracelulares producidas por bacterias marinas se incluyen amilasas, glucamilasas, glucosaisomerasas, pectinasas (Stanley \& Stanley, 1986) y otras como agarasas, quitinasas, alginasas, lipasas, DNasas, esterasas y proteasas (Fenical \& Jensen, 1993; León et al., 2000). En la actualidad, las bacterias productoras de proteasas han adquirido especial relevancia, ya que pue- 
den ser utilizadas en procesos productivos como la industria de los detergentes, alimentos y bebidas, así como la clarificación de cerveza fría y otras bebidas, ablandamiento de carnes rojas, industria del cuero, entre otras (Schmidt, 1981).

Asimismo, la tecnología enzimática microbiana ocupa un papel importante dentro de la biotecnología, específicamente en el sector alimentario, farmacéutico y salud. Alrededor del $65 \%$ de las enzimas que se producen industrialmente están de una u otra manera relacionadas con la industria alimentaria. Sólo las proteasas alcalinas empleadas en la industria de detergentes ocupan el $25 \%$ del total, las restantes corresponden a aplicaciones en las áreas farmacéuticas, industrial y analítica (Bárzana \& López-Murguía, 1995).

Una de las alternativas viables para mejorar la eficiencia, reducir los costos y aumentar la disponibilidad de algunas enzimas de interés industrial, es el de buscar y seleccionar microorganismos con altas capacidades de producción a partir de ambientes marinos. Asimismo, es de suma importancia evaluar sus condiciones óptimas de crecimiento, producción, actividades enzimáticas y caracterización fenotípica.

El presente estudio tuvo como finalidad aislar y seleccionar bacterias con capacidad de producir proteasas extracelulares de interés o aplicación biotecnológica procedentes de zonas marinas contaminadas con efluentes pesqueros.

\section{Materiales y métodos}

\section{Muestras de efluentes}

Las bacterias fueron aisladas de efluentes pesqueros emitidos por la fábrica del consorcio APROPISCO en Playa Lobería - Paracas, las fábricas pesqueras de Chancay (200 m al norte del muelle de Chancay) y efluentes del Terminal Pesquero de Ventanilla (Callao).

\section{Recolección de muestras}

Un total de 30 muestras de agua de mar contaminadas por efluentes pesqueros se co- lectaron en frascos estériles de $250 \mathrm{~mL}$ de capacidad a una distancia entre 5 a 50 metros de la orilla. Las muestras fueron refrigeradas y transportadas al laboratorio de Microbiología Ambiental y Biotecnología de la Facultad de Ciencias Biológicas de la Universidad Nacional Mayor de San Marcos.

\section{Aislamiento de bacterias}

Las muestras colectadas fueron procesadas en el laboratorio realizando diluciones seriadas al décimo $\left(10^{-1}, 10^{-2} \ldots 10^{-5}\right)$ y utilizando como diluyente agua de mar filtrada y esterilizada. El aislamiento y el recuento de bacterias marinas se realizó según la metodología indicada por León et al. (2000), sembrando alícuotas de $0,1 \mathrm{~mL}$ de las diluciones en Agar Marino (AM) 2216 (Difco), a pH 8,0, e incubados a $25^{\circ} \mathrm{C}$ por 5 días.

\section{Elección de cepas proteolíticas}

Para determinar la capacidad proteolítica de las cepas se tomaron $10 \mathrm{~mL}$ de los cultivos de $48 \mathrm{~h}$ y se repicaron en AM suplementado con caseína al 1\%, pH 8,0. Los cultivos fueron incubados a $25^{\circ} \mathrm{C}$ hasta por 5 días. La capacidad hidrolítica de las cepas se reconoció mediante la formación de halos transparentes alrededor de las colonias; los cuales se midieron cada 24 horas hasta por un periodo de 5 días. Las cepas fueron mantenidas en AM semisólido con adición de $10 \%$ de glicerol para realizar las pruebas experimentales posteriores.

\section{Optimización de los parámetros de cre- cimiento}

La optimización de los parámetros de crecimiento se realizó utilizando las cinco cepas seleccionadas signadas como CM43, CM44, CM45, CM46 y CM48; las cuales fueron sembradas en Caldo Marino (CM) suplementados con caseína $1 \%$ y sometidas al efecto de diferentes temperaturas $(5,8,15,25,37$ y 44 $\left.{ }^{\circ} \mathrm{C}\right), \mathrm{pH}(5,0 ; 6,0 ; 8,0 ; 9,0$ y 10,0$)$ y concentraciones crecientes de $\mathrm{NaCl}(0,3,6,9$ y $10 \%)$. Los cultivos en todos los casos fueron incubados hasta por 5 días. 


\section{Evaluación de la actividad proteolítica.}

Para estimular la actividad enzimática de las cepas aisladas, éstas se reactivaron en CM bajo las condiciones ya indicadas, y luego se sembraron $1 \mathrm{~mL}$ de estos cultivos $\left(10^{6} \mathrm{UFC} /\right.$ $\mathrm{mL}$ aproximadamente) en frascos conteniendo $100 \mathrm{~mL}$ de $\mathrm{CM}$ suplementados con caseína al $1 \%$, a pH 8,0 e incubados a $25^{\circ} \mathrm{C}$ con

Tabla 1. Comportamiento proteolítico de las 26 cepas de bacterias marinas seleccionadas según el tamaño de los halos de actividad ( $\mathrm{mm}$ de diámetro) en relación al tiempo de incubación.

\begin{tabular}{|c|c|c|c|c|c|}
\hline CEPAS & $24 \mathrm{~h}$ & $48 \mathrm{~h}$ & $72 \mathrm{~h}$ & 96h & $120 \mathrm{~h}$ \\
\hline CM3 & + & H & H+ & $\mathrm{HH}$ & H+ \\
\hline CM5 & + & H & H+ & $\mathrm{HH}$ & H+ \\
\hline CM9 & + & H & H+ & $+1+$ & H+ \\
\hline CM 16 & \pm & + & + & + & $H$ \\
\hline CM 20 & \pm & + & H & H & + \\
\hline CM22 & \pm & + & H & H & + \\
\hline CM26 & - & + & + & + & H+ \\
\hline CM 34 & - & - & + & + & $H$ \\
\hline CM 39 & - & - & + & + & + \\
\hline CM42 & - & - & + & + & + \\
\hline CM43 & - & + & + & + & + \\
\hline CM 44 & \pm & + & H+ & $H$ & H+ \\
\hline CM45 & - & - & + & $+1+$ & H+ \\
\hline CM46 & - & - & + & + & $H$ \\
\hline CM 48 & + & H & H+ & +11+ & H+H \\
\hline CM58 & \pm & \pm & + & + & + \\
\hline CM61 & \pm & \pm & + & + & + \\
\hline CM63 & \pm & \pm & + & H & + \\
\hline CM 78 & \pm & \pm & + & H & + \\
\hline CM80 & - & \pm & + & H & + \\
\hline CM85 & - & \pm & + & H & + \\
\hline CM87 & \pm & \pm & + & + & + \\
\hline CM95 & \pm & \pm & + & + & + \\
\hline CM98 & \pm & \pm & + & + & + \\
\hline CM 112 & - & \pm & + & + & + \\
\hline CM 115 & - & \pm & + & + & + \\
\hline
\end{tabular}

Tamaño de los halos de actividad:

- $\quad$ : no se evidencia halo

$\pm \quad$ : tamaño del halo < a $2 \mathrm{~mm}$

$+\quad$ : aproximado a $10 \mathrm{~mm}$

$+\quad$ : aproximado a $15 \mathrm{~mm}$

+++ : aproximado a $20 \mathrm{~mm}$

++++ : aproximado a $25 \mathrm{~mm}$ agitación constante de $120 \mathrm{rpm}$ hasta por 96 horas. La actividad enzimática $(\mathrm{U} / \mathrm{mL})$ y actividad específica (U/mg de proteína) de las cepas fueron determinadas según la metodología recomendada por Nieto y Ellis (1986) y Takahashi y Osaka (1970). Para ello, se tomaron los sobrenadantes de cultivos obtenidos luego de una centrifugación a $2500 \mathrm{rpm}$ por 10 minutos a $4{ }^{\circ} \mathrm{C}$. Estas pruebas se ejecutaron cada $24 \mathrm{~h}$ hasta completar las $96 \mathrm{~h}$. La mezcla de reacción se preparó en tubos conteniendo $1 \mathrm{~mL}$ de la solución de caseína (Difco) al 1\%, pH 8,0 y agregando alicuotas de $0,2,0,5$ y $1 \mathrm{~mL}$ de los sobrenadantes. La mezcla se incubó en baño maría por 20 minutos a $25^{\circ} \mathrm{C}$ agitando suavemente y se detuvo dicha reacción adicionando $2 \mathrm{~mL}$ de Ácido Tricloroacético (ATC) al 10\% (p/v); luego se centrifugó a $2500 \mathrm{rpm}$ por 10 minutos a temperatura ambiente, y a cada sobrenadante se le midió su Densidad Óptica a 280 nm comparándose los resultados con la curva estándar del aminoácido tirosina. Se realizó el mismo experimento con las cinco cepas en estudio.

La unidad de actividad fue definida como la cantidad de enzima que libera un micromol de aminoácidos por minuto por $\mathrm{mL}$ o miligramo de proteína. Por otro lado, el contenido proteico de los extractos obtenidos se realizó por el método de Lowry empleando el reactivo Folin Ciocalteau (Sigma) y Seroalbúmina de bovino (mg/mL) (Sigma) como estándar.

\section{Caracterización fenotípica de las bacte- rias aisladas}

La caracterización fenotípica de las cepas CM43, CM44, CM45, CM46 y CM48 se realizó mediante métodos convencionales en tubos y placas. Como medio base para todas las pruebas se utilizó el CM o AM a pH 8,0. La temperatura de incubación fue en todos los casos a 25 ${ }^{\circ} \mathrm{C}$ hasta por 5 días. Las cepas en estudio fueron sometidas a observaciones microscópicas y pruebas conducentes a la caracterización morfológica, fisiológica y bioquímica según procedimientos descritos por Baumman et al. (1972), Oliver (1982), Ortigoso et al. (1994). 


\section{Resultados y discusión}

\section{Cepas con actividad proteolítica}

El comportamiento proteolítico de las 26 cepas aisladas a partir de muestras de agua de mar contaminadas con efluentes pesqueros se muestran en la Tabla 1. Como se puede apreciar, es la cepa CM48 la que presentó el mayor halo de actividad (tamaño aproximado de $25 \mathrm{~mm}$ de diámetro) luego de 96 horas de incubación a $25{ }^{\circ} \mathrm{C}$; sin embargo, el comportamiento de las demás cepas es algo homogéneo. Estos resultados son coincidentes con los reportados por otros autores como Marty \& Martín (1992) y Harris (1993), quienes analizaron la actividad enzimática de bacterias ais-

Tabla 2. Efecto de los parámetros fisico-químicos en el crecimiento de 5 cepas de bacterias seleccionadas por su mayor actividad proteolítica sobre caseína al 1\%. En todos los casos los cultivos fueron incubados hasta por 5 días.

\begin{tabular}{|c|c|c|c|c|c|}
\hline Parár & $\begin{array}{l}\text { CM } 43 \\
s\end{array}$ & CM 44 & $\begin{array}{l}\text { Cepas } \\
\text { CM } 45\end{array}$ & CM 46 & CM 48 \\
\hline \multicolumn{6}{|c|}{$\mathrm{NaCl}(\%)$} \\
\hline 0 & + & + & + & ++ & - \\
\hline 3 & + & + & + & + & + \\
\hline 6 & + & + & + & + & + \\
\hline 9 & + & + & + & + & + \\
\hline 10 & + & - & + & - & + \\
\hline \multicolumn{6}{|l|}{ pH: } \\
\hline 5,0 & + & + & + & + & + \\
\hline 6,0 & + & + & + & + & + \\
\hline 8,0 & + & + & + & + & + \\
\hline 9,0 & + & + & + & + & + \\
\hline 10,0 & + & + & + & + & + \\
\hline \multicolumn{6}{|c|}{ Temp. ( $\left.{ }^{\circ} \mathrm{C}\right)$} \\
\hline 5 & - & - & - & - & - \\
\hline 8 & + & + & + & + & + \\
\hline 15 & + & + & + & + & + \\
\hline 25 & + & + & + & + & + \\
\hline 37 & + & + & $H$ & + & 0 \\
\hline 44 & - & + & + & + & + \\
\hline
\end{tabular}

ladas de varias especies de invertebrados acuáticos, demostrando que una gran proporción de crustáceos y moluscos estudiados eran portadores de bacterias con actividad multienzimática principalmente proteolítica. Por su parte, León et al. (2000) en estudios realizados sobre la microbiota bacteriana asociada a invertebrados marinos de la costa central peruana encontraron que el $62,74 \%$ de las cepas aisladas mostraban actividad proteolítica sobre la caseína, sugiriendo la posibilidad de utilizar cepas nativas con propósitos biotecnológicos. Ramírez et al. (1999), evaluaron la actividad proteolítica extracelular de levaduras marinas aisladas en Agar Papa Dextrosa enriquecida con caseína al 1\%; sin embargo, las pruebas cualitativas de la actividad enzimática de 5 cepas seleccionadas mostraron halos de actividad inferiores a $11 \mathrm{~mm}$ de diámetro, en tanto la mayoría de las cepas descritas en el presente trabajo en muchos casos superan los $20 \mathrm{~mm}$ de diámetro.

\section{Determinación de los parámetros óptimos de crecimiento}

Los resultados de la evaluación de las cinco cepas frente a diferentes parámetros físico-químicos, se muestran en la Tabla 2. Se observa que la mayoría de las cepas crecen en un amplio rango de cloruro de sodio incluyendo la ausencia de esta sal en el medio (CM43, CM44, CM45 y CM46). Debido a este comportamiento las bacterias de este tipo han sido consideradas por otros autores, como posibles cepas alóctonas que se adaptaron a las condiciones marinas, tal vez bacterias de aguas continentales o de origen terrestre (Ronald \& Bartha, 1998). Las cepas CM43, CM45 y CM48 tuvieron un buen crecimiento en medios hasta con $10 \%$ de $\mathrm{NaCl}$. Asimismo, todas las cepas tuvieron buen crecimiento en el rango de 3 a $9 \%$ de $\mathrm{NaCl}$, excepto la cepa CM48, la cual no creció en ausencia de $\mathrm{NaCl}$, considerándose por lo tanto como una cepa autóctona de ambientes marinos. Onishi et al. (1965), realizaron estudios en ambientes marinos encontrando a bacterias proteolíticas 


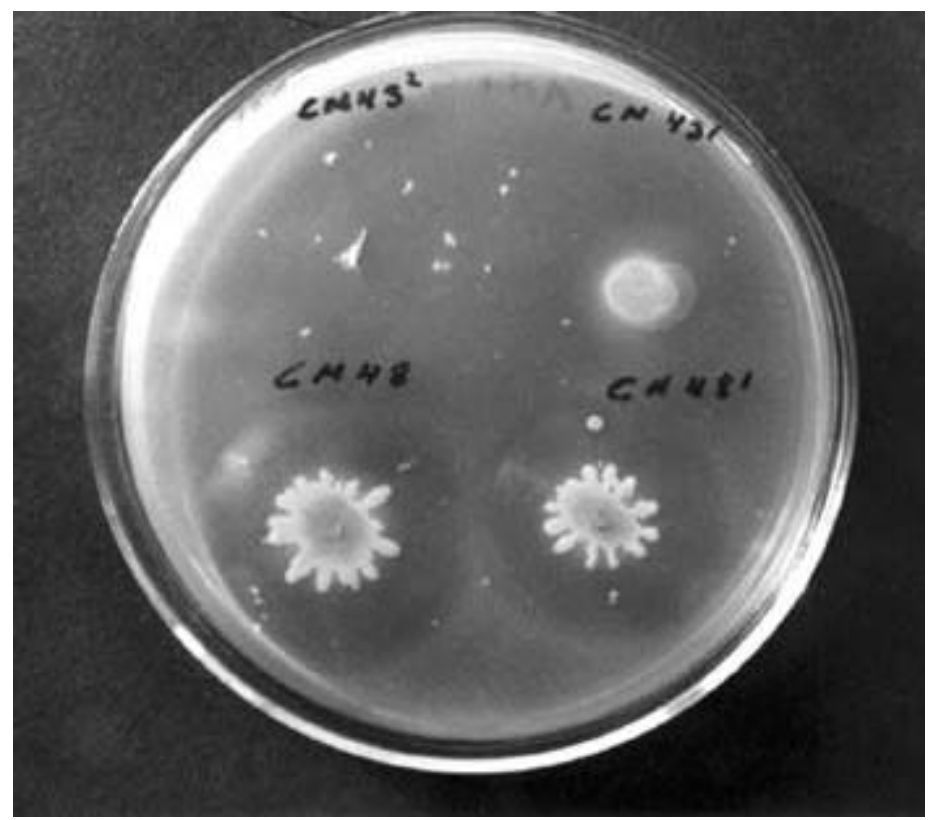

Figura 1. Actividad proteolitica de la cepa CM48 en AM suplementado con caseína al $1 \%$. Obsérvese el halo transparente alrededor de la colonia. Cultivo de $96 \mathrm{~h}$.

halotolerantes inclusive a altas concentraciones de $\mathrm{NaCl}$ (3-20\%), con un crecimiento óptimo a $14 \%$ de $\mathrm{NaCl}$, siendo las bacterias dominantes pertenecientes a los géneros Pseudomonas y Acinetobacter y un menor porcentaje a Flavobacterium y Caulobacter y algunos Gram positivos como Bacillus y Micrococcus.

Las cepas evaluadas frente a diferentes valores de $\mathrm{pH}$ mostraron buen crecimiento en el rango de 5,0 a 10,0; demostrando así su comportamiento moderadamente alcalófilo propio de las bacterias de ecosistemas marinos. En cuanto al perfil térmico, cuatro de ellas (CM44, CM45, CM46 y CM48) crecieron óptimamente entre 8 a $44{ }^{\circ} \mathrm{C}$, excepto la cepa $\mathrm{CM} 43$ que tuvo como temperatura límite a $37^{\circ} \mathrm{C}$. Ningu- na de las cepas creció a $5{ }^{\circ} \mathrm{C}$. En relación con nuestros resultados, Vázquez et al. (1999) aislaron 8 cepas de Pseudomonas sp de biotopos costeros antárticos, productoras de proteasas con una actividad óptima a $25^{\circ} \mathrm{C}$ a pesar de haber sido aisladas de ambientes con temperaturas bajas. Estas temperaturas de crecimiento son características de los microorganismos c onsiderados como psicrotróficos o psicrófilos moderados.

\section{Evaluación de la actividad proteolítica}

La evaluación de la actividad proteolítica de las 5 cepas en estudio, demostró que la cepa CM48 perteneciente al género Pseudomonas sp. fue la que presentó mejor actividad enzimática frente a la caseína al $1 \%$ y a diferentes volúmenes de los crudos enzimáticos.

Tabla 3. Actividad Enzimática $(\mathrm{U} / \mathrm{mL})$ y Actividad Específica $(\mathrm{U} / \mathrm{mg})$ de los crudos enzimáticos de cultivos de 5 cepas de bacterias proteolíticas sometidas a diferentes tiempos (h) de incubación.

\begin{tabular}{lcccccccc}
\hline & \multicolumn{2}{c}{ 24h } & \multicolumn{2}{c}{ 48h } & \multicolumn{2}{c}{ 72h } & \multicolumn{2}{c}{ 96h } \\
Cepas & $\mathbf{U} / \mathbf{m L}$ & $\mathbf{U} / \mathbf{m g}$ & $\mathbf{U} / \mathbf{m L}$ & $\mathbf{U} / \mathbf{m g}$ & $\mathbf{U} / \mathbf{m L}$ & $\mathbf{U} / \mathbf{m g}$ & $\mathbf{U} / \mathbf{m L}$ & $\mathbf{U} / \mathbf{m g}$ \\
\hline CM 43 & 3 & 11,6 & 4 & 11,64 & 7 & 12,02 & 7 & 12,17 \\
CM 44 & 4 & 9,73 & 4 & 9,75 & 4 & 10,07 & 5 & 9,98 \\
CM 45 & 2 & 8,57 & 3 & 9,76 & 7 & 12,09 & 7 & 12 \\
C M 46 & 2 & 9,32 & 3 & 9,6 & 4 & 10,1 & 4 & 9,81 \\
CM 48 & 4 & 13,7 & 6 & 15,43 & 10 & 17,38 & 10,37 & 17,76 \\
\hline
\end{tabular}


Tabla 4. Caracterización fenotípica de 5 cepas de bacterias con actividad proteolítica sobre caseína al 1\%, aisladas de agua de mar contaminadas con efluentes pesqueros.

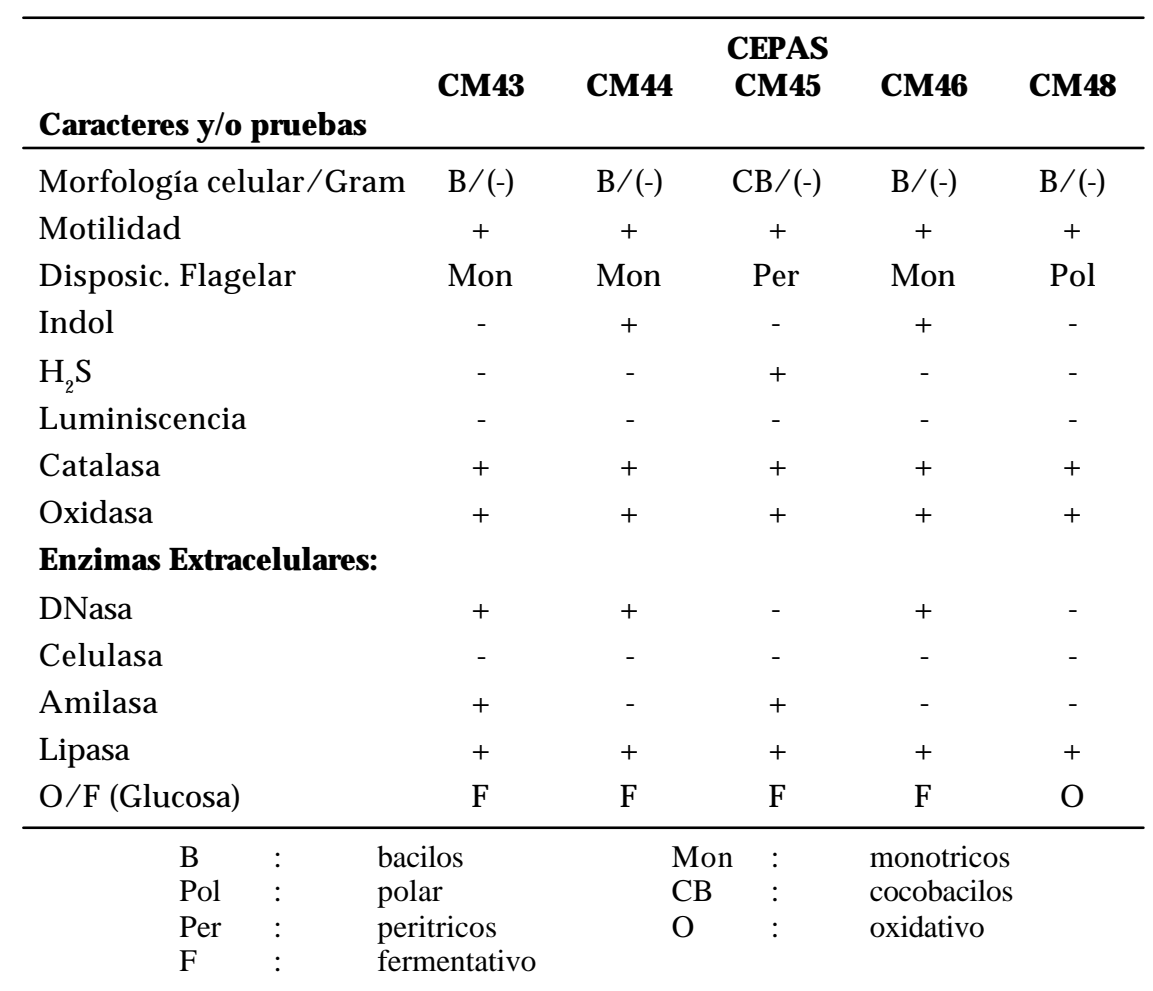

Asimismo, en evaluaciones posteriores con crudos tomados de cultivos de diferentes tiempos de incubación (desde 24 hasta 96 h) las actividades enzimáticas más altas las registró la cepa CM48, con actividades enzimáticas de 9,91 y de $10,37 \mathrm{U} / \mathrm{mL}$ y actividades específicas de 17,38 y $17,76 \mathrm{U} / \mathrm{mg}$ con crudos enzimáticos de 72 y 96 horas de incubación respectivamente (Tabla 3 y Fig. 1). Al respecto, Velásquez et al. (1999) también encontraron valores similares de actividad proteolítica de 33,65 U/mL, aunque dichos autores emplearon una cepa de Flavobacterium indolegenes cultivadas en medio Horikoshi (inductor de proteasas) en agitación constante de $200 \mathrm{rpm}$ y $30{ }^{\circ} \mathrm{C}$ de incubación, durante 72 horas. Por otro lado Vázquez et al. (1999) reportaron la actividad proteolítica de 8 cepas de Pseudomonas aisladas de biotopos costeros antárticos. Las proteasas mostraron actividades de $1700 \mathrm{U} / \mathrm{L}$ a $1 \times 10^{5} \mathrm{U} / \mathrm{L}$ en extractos de cultivos de 48 a $72 \mathrm{~h}$ a $20{ }^{\circ} \mathrm{C}$. Dichos autores no presentan resultados de actividad específica. Por otro lado, Ramírez et al. (1999), evaluaron también la actividad proteolítica de crudos enzimáticos producidos por levaduras marinas de los géneros Pichia y Debaromyces cultivados en el medio Papa Dextrosa con caseína al $1 \%$ e incubados a temperatura ambiente por 4 días. Los resultados de esta evaluación, a pesar que en nuestro caso no se trabajó con levaduras, son de alguna manera comparables a los halos presentados por nuestras cepas luego de 4 días de incubación en medio AM suplementado con caseína al $1 \%$, siendo la cepa CM48 una Pseudomonas sp. la que demostró mayor actividad, con un diámetro superior a $20 \mathrm{~mm}$ de diámetro a las 96 horas de incubación a $25^{\circ} \mathrm{C}$ y a un pH de 8,0 (Tabla 1). La actividad proteolítica de 5 cepas marinas (CM43, CM44, CM45, CM46 y CM48) comparadas con otros trabajos mencionados resultan ser interesantes para continuar los estudios relacionados a mejorar las 
condiciones de crecimiento y producción de enzimas proteolíticas extracelulares en microorganismos marinos.

\section{Caracterización fenotípica}

La caracterización fenotípica de las 5 cepas de bacterias previamente seleccionadas se muestra en la Tabla 4. Todas ellas resultaron tener formas bacilares o cocobacilares, Gram negativas, móviles, flagelares, catalasa y oxidasa positivas. De acuerdo a las pruebas de caracterización morfológica, fisiológica y bioquímica las cepas en estudio pertenecen a los siguientes géneros: Aeromonas (cepas CM43, CM44 y CM46), Pseudomonas (cepa CM48) y Alcaligenes (cepa CM45). En gran parte nuestros resultados corroboran los hallazgos de otros autores (Vásquez et al., 1999; Velásquez et al., 1999; León y García-Tello, 1998; León et al., 2000), quienes trabajando con muestras procedentes de ambientes marinos tales como agua, organismos bentónicos, invertebrados de vida libre y en cultivos, lograron aislar bacterias con actividad proteolítica pertenecientes a los géneros Pseudomonas, Vibrio, Flexibacter, Moraxella, Bacillus y Flavobacterium. Otros autores como Ramírez et al. (1999), además de cepas bacterianas reportan también el hallazgo de levaduras marinas con actividad proteolítica sobre la caseína pertenecientes a los géneros Pichia y Debaromyces.

\section{Conclusiones}

Las bacterias marinas productoras de proteasas y otras exoenzimas pueden presentar mejores ventajas que las fuentes actuales utilizadas en la industria, ya que dichas cepas presentan un buen crecimiento a bajas temperaturas, valores de $\mathrm{pH}$ variables $(5,0-10,0)$ y un rango amplio de concentraciones de cloruro de sodio. En este sentido, las bacterias marinas son ideales para ser utilizadas en la producción de enzimas de importancia industrial; asimismo, ofrecen alternativas en el tratamiento de aguas contaminadas con efluentes pesqueros. Los resultados mostraron que la cepa con mayor actividad proteolítica correspondió al género Pseudomonas sp. considerada esta cepa como autóctona de ambientes marinos por crecer óptimamente en $\mathrm{pH}$ alcalino y requerir de condiciones halófilas y psicrófilas moderadas.

\section{Literatura citada}

Bárzana, E. y López-Murguía. 1995. La Tecnología Enzimática. En: Biotecnología Alimentaria,pp. 103-123. Limusa, México D.F.

Baumann, L.; M. Baumann, M. Mandel and R. Allen, 1972. Taxonomy of aerobic marine Eubacteria. J. Bacteriol 110: 402-429.

Feller, G.; E. Narinx, J. A. Arpingny, M. Haleb, E. Baise, S. Genicot and Ch. Gerday. 1996. Enzymes from psychrophylic organism. FEMS Microbiol. Rev. 18: 189-202.

Fenical, W. and PR. Jensen. 1993. Marine microorganisms: a new biomedical resource. In: Marine Biotechnology. Pharmaceutical and Bioactive Natural Products. Ed. D. H. Attaway, O.R. Zaborsky. New York Plenum Press, 1:419-457.

Harris, J.M. 1993. The presence, nature and role of gut microflora in aquatic invertebrates: a synthesis. Microb. Ecol. 25: 195-231.

León, J. y P. García -Tello. 1998. Cepas Nativas del Bacteriomeuston Marino y su Actividad inhibitoria de Bacterias Ictiopatogenas. Rev. peru. biol. 5(1): 47-64.

León, J.; F. Pellón, V. Unda, J. David, C. Anaya, y V. Mendoza. 2000. Producción de enzimas extracelulares por bacterias aisladas de invertebrados marinos. Rev. Peru. Biol. 7(2): 202-210.

Marty, P. \& Y. Martín. 1992. Aerobic heterotrophic bacteria associated with some Mediterranean coastal benthic invertebrates: characterization of strains, exoenzyme and antibiotic production. Mar Life 1(1): 1-8.

Nieto, T. \& A. Ellis. 1986. Characterization of extracelular metallo and serine proteases of Aeromonas hydrophila. Gem. Microbiol. 132: 1975-1979.

Oliver, J. D. 1982. Taxonomic Scheme for the identification of marine bacteria. Deep-Sea Res. 29: 795-798.

Onishi, H; E. Mac Cane \& E. Gibbons. 1965. A synthetic medium for extremely halophilic bacteria. Can. J. Microbiol. 11: 365-373

Ortigoso, M.; E. Garay \& M-J. Pujalte. 1994. Numerical Taxonomy of aerobic, Gram - negative bacteria associated with oysters and surrounding seawater of the Mediterranean coast System. Appl. Microbiol. 17: 589-600

Ramírez, O.; N. Hernández \& J. Ochoa. 1999. Aprovechamiento biotecnológico de levaduras mari- 
nas, Memorias del IV Congreso Latinoamericano de Biotecnología y Bioingeniería. P.III. 68. México.

Ronald, M. \& R. Bartha. 1998. Ecología microbiana y microbiología ambiental, 4ta. Edición. Edit. Pearson Educación, S. A. Madrid, España.

Stanley, I. T. \& P. M. Stanley. 1986. Potential commercial applications in aquatic microbiology. Microb. Ecol., 12: 79-100.

Schmidt, H. 1981. Avances en ciencias y tecnología de los alimentos. Alfabeta Impresora, Santiago de Chile.

Takahashi, T. \& A. Osaka. 1970. Purification and characterization of a protein in venom of trimeresurus flavoviridis, complete separation of the enzyme from hemorrhagie activity. Biochem. Biophys. Acta. 198:293.
Vázquez, S.; W. Mac Cormack \& E. Fraile. 1999. Caracterización de proteasas producidas por bacterias psicrotróficas antárticas, IV Congreso Latinoamericano de Biotecnología y Bioingeniería. P IV. 8. México.

Velásquez, R.; A. Monroy \& R. Oliart. 1999. Purificación y caracterización parcial de las proteasas alcalinas producidas por Flavobacterium indolegenes. IV Congreso latinoamericano de Biotecnología y Bioingeniería P.I.49-México. 